\title{
Acute Kidney Injury and Mortality in Hospitalized Patients
}

\author{
Henry E. Wang ${ }^{\text {a }}$ Paul Muntner ${ }^{\text {b, c }}$ Glenn M. Chertow ${ }^{d}$ David G. Warnock ${ }^{c}$ \\ Departments of ${ }^{a}$ Emergency Medicine and ${ }^{b}$ Epidemiology, and ${ }^{c}$ Division of Nephrology, Department of Medicine, \\ University of Alabama at Birmingham, Birmingham, Ala., and d Division of Nephrology, Department of Medicine, \\ Stanford University, Stanford, Calif., USA
}

\section{Key Words}

Acute kidney injury $\cdot$ Chronic kidney disease $\cdot$ Mortality • Health services

\begin{abstract}
Background: The objective of this study was to determine the incidence of acute kidney injury (AKI) and its relation with mortality among hospitalized patients. Methods: Analysis of hospital discharge and laboratory data from an urban academic medical center over a 1-year period. We included hospitalized adult patients receiving two or more serum creatinine $(\mathrm{s} C r)$ measurements. We excluded prisoners, psychiatry, labor and delivery, and transferred patients, 'bedded outpatients' as well as individuals with a history of kidney transplant or chronic dialysis. We defined AKI as (a) an increase in $\mathrm{sCr}$ of $\geq 0.3 \mathrm{mg} / \mathrm{dl}$; (b) an increase in $\mathrm{sCr}$ to $\geq 150 \%$ of baseline, or (c) the initiation of dialysis in a patient with no known history of prior dialysis. We identified factors associated with AKI as well as the relationships between AKI and in-hospital mortality. Results: Among the 19,249 hospitalizations included in the analysis, the incidence of AKI was $22.7 \%$. Older persons, Blacks, and patients with reduced baseline kidney function were more likely to develop AKI (all $p<$ 0.001). Among AKI cases, the most common primary admit-
\end{abstract}

ting diagnosis groups were circulatory diseases (25.4\%) and infection (16.4\%). After adjustment for age, sex, race, admitting $\mathrm{s} C r$ concentration, and the severity of illness index, AKI was independently associated with in-hospital mortality (adjusted odds ratio 4.43, 95\% confidence interval 3.685.35). Conclusions: AKI occurred in over 1 of 5 hospitalizations and was associated with a more than fourfold increased likelihood of death. These observations highlight the importance of AKI recognition as well as the association of AKI with mortality in hospitalized patients.

Copyright $\odot 2012$ S. Karger AG, Basel

\section{Introduction}

Acute kidney injury (AKI) is a syndrome characterized by decreased glomerular filtration. The spectrum of AKI ranges from minimal elevations in serum creatinine $(\mathrm{sCr})$ to complete anuric kidney failure. Despite ample knowledge of the biologic basis of AKI, descriptions of the incidence, risk factors, sequelae, and outcomes of AKI remain relatively limited or have been based upon older descriptions without reflecting the most current definitions or practice patterns. For example, studies of $\mathrm{sCr}$ trends at single centers in Boston, Mass., USA, Austin,

\section{KARGER}

Fax +4161306 1234

E-Mail karger@karger.ch

www.karger.com
(C) 2012 S. Karger AG, Basel

0250-8095/12/0354-0349\$38.00/0

Accessible online at:

www.karger.com/ajn
Henry E. Wang, MD, MS

Department of Emergency Medicine

University of Alabama at Birmingham

619 19th Street South, OHB 251, Birmingham, AL 35249 (USA)

Tel. +1 205996 6526, E-Mail hwang@uabmc.edu 
Australia, and Chicago, Ill., USA, used hospital data that were more than 10 years old [1-3]. Other efforts identified AKI using hospital discharge diagnosis codes $[4,5]$. Many studies have focused on AKI in intensive care units and have not considered AKI in the general hospital setting [6-12].

The evolving definitions and care recommendations for AKI underscore the need for updated epidemiologic data describing this condition [7]. These observations could help to highlight the current burden of AKI, the affected patient groups, the involved care settings as well as the current risk of mortality and could guide efforts to identify cases or target therapies. The objective of this study was to characterize the current incidence of AKI in patients hospitalized at an urban academic medical center and to assess the association of AKI with inpatient mortality.

\section{Methods}

Design

We analyzed hospital discharge data linked with clinical laboratory results for a single year from an urban academic tertiary referral medical center. This study was approved by the Institutional Review Board of the University of Alabama at Birmingham (UAB).

\section{Setting and Source of Data}

The UAB Hospital is an urban academic tertiary care referral medical center located in Birmingham, Ala., USA. The 908-bed institution contains an emergency department with 64,000 visits per year, is the only level I trauma center in Alabama, and has 8 intensive care units containing more than 180 critical care beds.

For this study, we used the hospital's standard discharge data set, consisting of patient demographics and diagnostic and financial information in the standard UB-04 format for the period of October 1, 2009 through September 30,2010. Data analysts linked the hospital data set with $\mathrm{sCr}$ concentrations obtained for each patient. The hospital used commercial databases to manage discharge and laboratory data (HealthQuest Data Systems, Highland, Calif., USA, and Cerner PathNet, Cerner, Inc., Kansas City, Mo., USA).

\section{Selection of Subjects}

We studied adult ( $\geq 18$ years old) patients hospitalized at the UAB Hospital between October 1, 2009 and September 30, 2010. We excluded prisoners, patients admitted to the psychiatry service or labor and delivery service, and patients transferred from other hospitals. We also excluded patients classified as 'bedded outpatients', a term used by the hospital to identify individuals admitted for a short term after scheduled surgical or other procedures. Because we sought to identify the development of AKI after presentation to the hospital, we excluded individuals with a history of end-stage renal disease requiring either kidney transplant (identified through discharge diagnosis ICD-9 V42.0) or mainte- nance dialysis (ICD-9 V45.1, V45.11, V45.12, V56, V56.0, V56.8). Because the analysis relied upon the comparison of serial sCr values, we included only patients with two or more sCr measurements.

\section{Definition of $A K I$}

Using definitions established by the Acute Kidney Injury Network (AKIN), we defined AKI as (a) an increase in $\mathrm{sCr}$ of $\geq 0.3$ $\mathrm{mg} / \mathrm{dl}$; (b) an increase in sCr to $\geq 150 \%$ of baseline, or (c) the initiation of dialysis in a patient with no known history of prior dialysis (ICD-9p 39.95, 54.98) [7]. Following Chertow et al. [1], we defined the baseline sCr as the lowest of the first three sCr levels for each patient. We characterized graded increases in AKI severity using categories established by the AKIN (stage $1=$ increase in sCr of $\geq 0.3 \mathrm{mg} / \mathrm{dl}$, or increase in $\mathrm{sCr}$ to $\geq 150-199 \%$ of baseline; stage $2=$ increase in sCr to $\geq 150-199 \%$ of baseline, and stage $3=$ increase in sCr to $\geq 300 \%$ of baseline or initiation of renal replacement therapy). Urine output measurements were not available in the data set and were not included in the definition of AKI used in this analysis. We did not include time parameters in the definition of AKI.

We used patient demographics (classifying race as black or non-black) and the Chronic Kidney Disease Epidemiology Collaboration (CKD-EPI) equation to calculate the estimated glomerular filtration rate (eGFR) [13]. We based eGFR upon the lowest of the first three sCr levels of each patient. We adjusted for the severity of illness using the risk adjustment methodology from the University Health System Consortium (UHC) clinical database [14]. Based upon the proprietary All Patients Refined Diagnosis Related Group (APR-DRG, 3M, Inc., St. Paul, Minn., USA), the UHC expected mortality index aggregates acuity data across diagnosis groups to generate a global expected mortality estimate for each hospitalization.

To avoid detecting AKI associated with protracted hospitalizations, we limited the study to the first 21 days of hospitalization after the first sCr measurement, as well as the first $60 \mathrm{sCr}$ measurements for each admission. Post hoc analysis confirmed that this strategy preserved $89.2 \%$ of the sCr measurements and $99.5 \%$ of the peak sCrvalues.

\section{Data Analysis}

We compared the characteristics of the patients with and without AKI using the $\chi^{2}$ test for discrete variables and Student's t test or the Wilcoxon rank-sum test for continuous variables, as appropriate. We compared the primary discharge diagnoses of cases with and without AKI, grouping the discharge diagnoses according to major ICD-9 diagnostic categories. Because AKI often complicates serious infections, we determined the incidence of AKI for patients with a primary infection diagnosis, using an infection taxonomy described by Angus et al. [15] and Wang et al. [16].

Using multivariable logistic regression, we determined the association between AKI and in-hospital mortality, fitting a series of models defining AKI in terms of absolute and relative increases in $\mathrm{sCr}$, and adjusting for age, sex, race, baseline sCr concentration, and natural $\log$ [UHC expected mortality index]. We defined stages of AKI using the AKIN system [7]. We repeated the analysis stratifying by the primary discharge diagnosis groups circulatory diseases (ICD-9 390-459.9), respiratory diseases (ICD-9 460-519.9), infections, injuries (ICD-9 800-950.9), and all other 
Fig. 1. Selection of the 19,249 cases included in the analysis. 'Bedded outpatients' refers to patients admitted to the hospital after scheduled operative procedures.

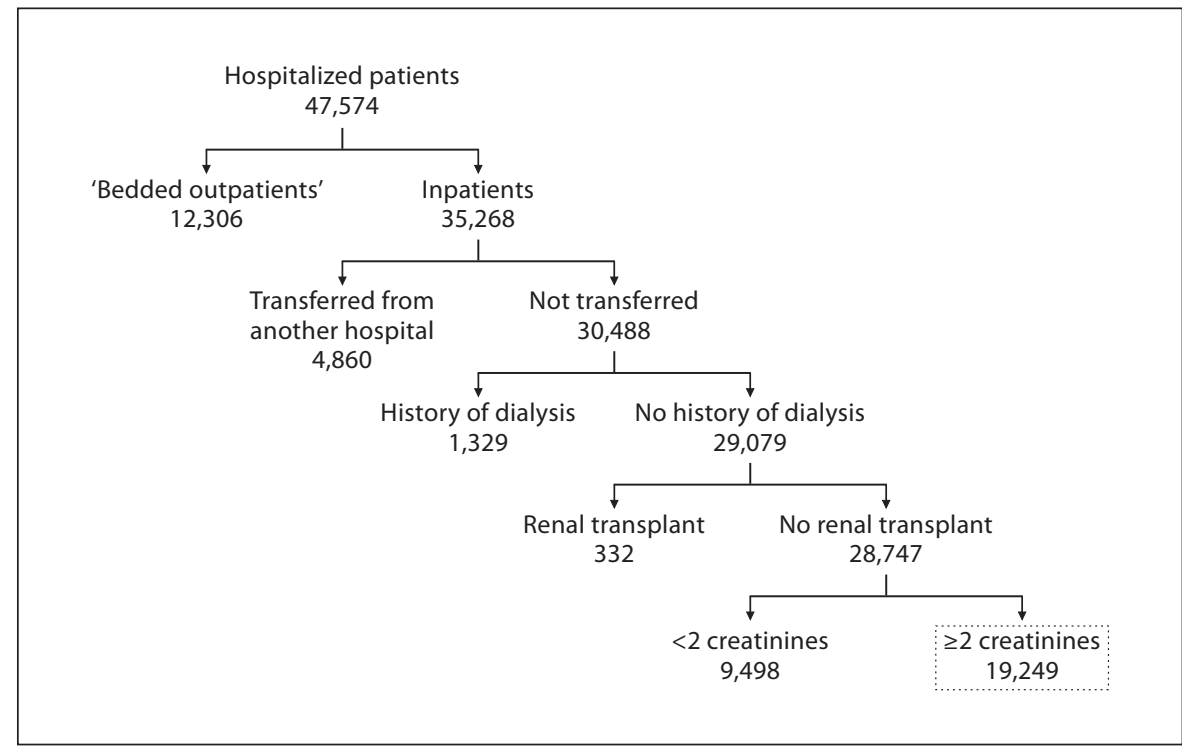

conditions $[15,16]$. In a sensitivity analysis, we repeated the mortality analysis including only the first hospitalization for each individual in the data set. We conducted all analyses using Stata version 12.0 (Stata Corp, College Station, Tex., USA).

\section{Results}

After excluding 'bedded outpatients', transferred patients, patients with a history of dialysis or kidney transplant, and those with one or no sCr determinations, we included 19,249 hospitalizations in the analysis (fig. 1). These 19,249 hospitalizations encompassed 15,096 individual patients; 1,612 individuals accounted for 4,153 repeat hospitalizations. Defining AKI as an increase in $\mathrm{sCr}$ of $\geq 0.3 \mathrm{mg} / \mathrm{dl}$, an increase in $\mathrm{sCr}$ to $\geq 150 \%$ of baseline, or the initiation of dialysis, the incidence of AKI was $22.7 \%$. The incidence of AKI by AKIN stage was: stage 1 , $15.8 \%$; stage $2,2.7 \%$, and stage $3,4.2 \%$.

\section{Characteristics of AKI Cases}

AKI was more likely in older and black individuals as well as in those with higher baseline $\mathrm{sCr}$ concentrations (table 1). AKI was less frequent among patients admitted through the emergency department than among other hospitalized patients. Almost $60 \%$ of patients developing AKI never received intensive care unit care during their hospitalization.

Among the AKI cases, the most common primary discharge diagnosis groups were circulatory diseases
(25.4\% of AKI) and infection (16.4\%) (online suppl. Appendix 1; for all online suppl. material, see www. karger.com/doi/10.1159/000337487). Complications of medical care (9.6\%), neoplasms (9.6\%), injury (8.1\%), and diseases of the respiratory system $(7.9 \%)$ comprised large portions of the patients who developed AKI during their hospitalization.

\section{Incidence and Correlates of Hospital-Acquired AKI}

The mortality rate for AKI was $10.8 \%$, compared to $1.5 \%$ for cases without AKI. Larger increases in sCr were associated with higher mortality rates: stage $1,6.3 \%$; stage $2,16.5 \%$, and stage $3,23.7 \%$. AKI was independently associated with in-hospital mortality after adjusting for demographic factors, baseline eGFR of $<60 \mathrm{ml} / \mathrm{min} /$ $1.73 \mathrm{~m}^{2}$, and UHC expected mortality score (table 2). Associations between AKI and mortality were present across major primary discharge diagnostic groups (table 3 ). When considering time to peak sCr (early: $\leq 7$ days vs. late: $>7$ days), AKI remained independently associated with mortality, but the odds ratio for death was higher among those with more prolonged hospitalizations and $\mathrm{sCr}$ peak values occurring after the seventh hospital day (table 4).

\section{Sensitivity Analysis}

When repeating the analysis including only the first hospitalization for each individual (i.e. excluding any cases that were re-admissions), we obtained similar estimates for the incidence of and adjusted mortality associated with AKI (online suppl. Appendix 2). 
Table 1. Characteristics of hospitalized patients with AKI

\begin{tabular}{|c|c|c|c|}
\hline Characteristic & $\begin{array}{l}\text { AKI } \\
(n=4,365)\end{array}$ & $\begin{array}{l}\text { No AKI } \\
(\mathrm{n}=14,884)\end{array}$ & $\begin{array}{l}\mathrm{p} \text { value } \\
\text { AKI vs. no AKI }\end{array}$ \\
\hline Age, years & $56.8 \pm 16.9$ & $54.6 \pm 17.7$ & $<0.001$ \\
\hline Race/ethnicity & & & $<0.001$ \\
\hline White & $2,529(57.9)$ & $9,613(64.6)$ & \\
\hline Black & $1,729(39.6)$ & $4,962(33.3)$ & \\
\hline Unknown & $8(0.2)$ & $33(0.2)$ & \\
\hline Baseline eGFR $<60 \mathrm{ml} / \mathrm{min} / 1.73 \mathrm{~m}^{2}$ & $1,555(35.6)$ & $2,752(18.5)$ & $<0.001$ \\
\hline Admission source & & & $<0.001$ \\
\hline Emergency department & $2,370(54.3)$ & $8,957(60.2)$ & \\
\hline Other & $1,995(45.7)$ & $5,927(39.8)$ & \\
\hline Admitted to ICU & $163(3.7)$ & $336(2.3)$ & $<0.001$ \\
\hline Peak sCr, mg/dl & $2.4 \pm 2.4$ & $1.1 \pm 0.8$ & $<0.001$ \\
\hline Maximum sCr increase, $\mathrm{mg} / \mathrm{dl}$ & $0.8 \pm 1.0$ & $0.1 \pm 0.1$ & $<0.001$ \\
\hline Time to peak sCr, days & $2.7(1.4-5.6)$ & $1.2(0.6-2.4)$ & $<0.001$ \\
\hline Hospital length of stay, days & $7(4-13)$ & $4(2-7)$ & $<0.001$ \\
\hline Hospital mortality & $470(10.8)$ & $223(1.5)$ & $<0.001$ \\
\hline
\end{tabular}

Values are $\mathrm{n}(\%)$, mean $\pm \mathrm{SD}$, or median (IQR). ICU $=$ Intensive care unit.

AKI based upon AKIN criteria and defined as (a) an sCr increase of $\geq 0.3 \mathrm{mg} / \mathrm{dl}$ from baseline, (b) an $\mathrm{sCr}$ increase to $\geq 150 \%$ of baseline, or (c) the initiation of dialysis [7].

Baseline sCr is defined as the lowest of the first three sCr measurements. Baseline eGFR calculated from baseline sCr using the CKD-EPI equation [13].

Table 2. Multivariable associations between AKI and all-cause mortality

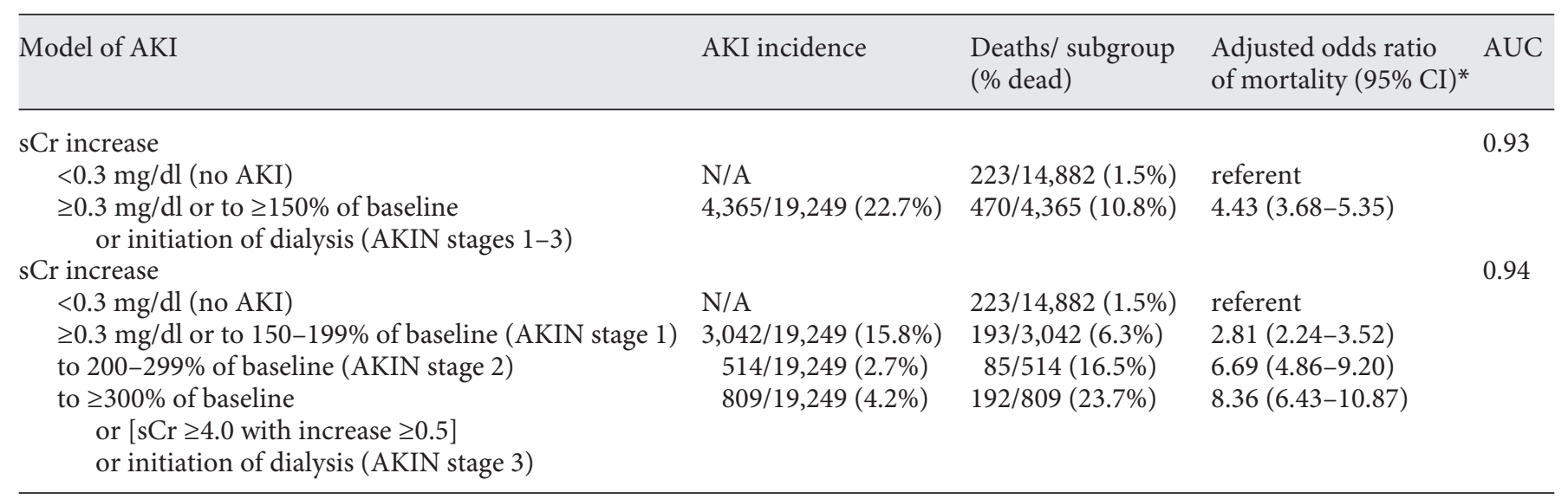

AKI stages based upon the AKIN staging system [7].

* Adjusted for age, sex, race (black/white/other), chronic kidney disease, and $\ln$ (UHC expected mortality).

$\mathrm{AUC}=$ Area under receiver-operating characteristic curve. 
Table 3. AKI and in-hospital all-cause mortality, stratified by primary discharge diagnosis

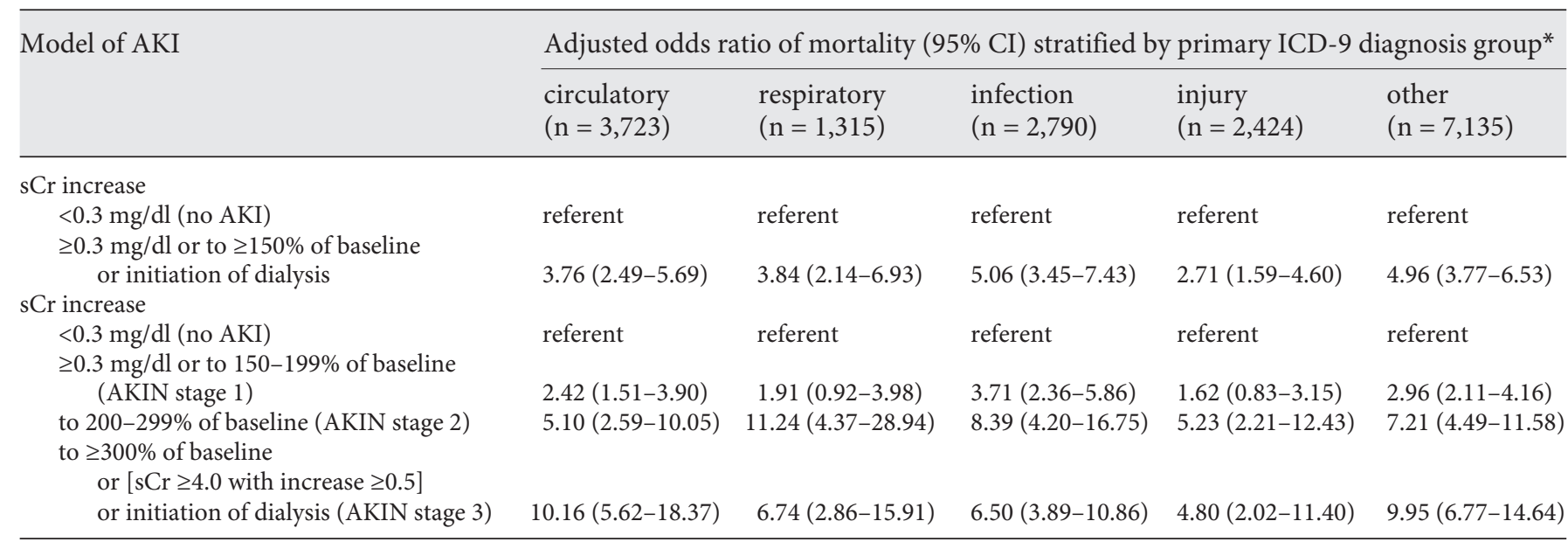

AKI stages based upon the AKIN staging system [7]. ICD-9 codes for each subgroup are listed in suppl. Appendix 1. ICD-9 codes for infection are based upon Angus et al. [15] and Wang et al. [16].

* Adjusted for age, sex, race (black/white/other), chronic kidney disease, and $\ln (\mathrm{UHC}$ expected mortality).

Table 4. Associations between AKI and all-cause mortality: early versus late sCr peak

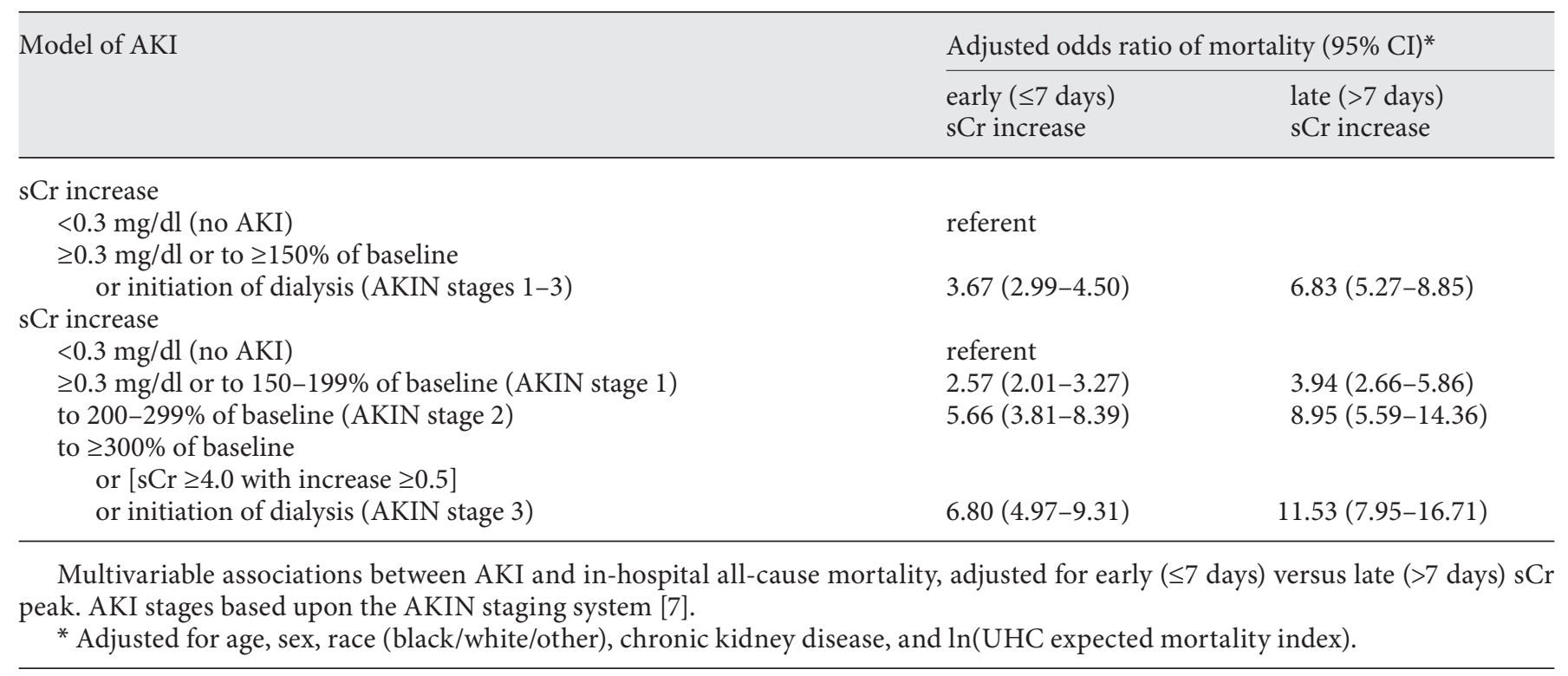

\section{Discussion}

We extend upon the findings of prior epidemiologic studies of AKI, confirming the high prevalence of AKI and the high mortality associated with the condition. Chertow et al. [1] examined AKI among roughly 9,000 in- patients in Boston, Mass., USA. We observed similar adjusted associations between AKI and hospital mortality despite the application of different risk adjustment techniques (DRG weights vs. UHC mortality index), definitions of chronic kidney disease (baseline sCr vs. eGFR), data from different academic institutions (Brigham and 
Women's Hospital vs. UAB Hospital), geographic regions (Boston, Mass., USA vs. Birmingham, Ala., USA), and time periods separated by over 12 years (September 1997 to April 1988 vs. October 2009 to September 2010) [13, 14].

The adequacy of risk adjustment is an inherent limitation of all observational studies. The current models appeared to demonstrate better discrimination (0.93) than that in Chertow et al. [1] (0.83), offering additional assurance that the observed associations with mortality are actually due to the independent effect of AKI rather than incomplete risk adjustment or unaccounted disease severity [1]. The UHC global expected mortality estimator is in use across the entire consortium, making direct comparisons of the association of AKI with mortality risk possible between different centers in the UHC.

The high incidence of AKI observed in our study is also consistent with other epidemiologic studies of AKI in the hospital setting. Creatinine-based studies of AKI in hospitalized patients in Austin, Australia, and Chicago, Ill., USA, utilized data that were more than 10 years old [2, 3]. Liangos et al. [4] and Waikar et al. [5] used national hospital discharge data to characterize the incidence of AKI but defined AKI using ICD-9 discharge diagnoses - not changes in serial $\mathrm{sCr}$ measurements. LaFrance and Miller [17] analyzed 1.2 million patient records from the Department of Veteran's Affairs (VA) Health Care System, identifying AKI rates ranging from 7.8 to $11 \%$. However, their patient population consisted mainly of men ( $95 \%$ males). Our study reflects the current demographic characteristics of AKI among inpatients at a major academic medical center. The similar findings between these studies also underscore the persistent incidence of AKI, despite heightened awareness and knowledge over the last decade. The observation that the association with inpatient mortality has not changed over this interval also emphasizes the importance of AKI in the hospital setting.

Our study offers additional key perspectives of AKI. Although we observed an AKI incidence of $21 \%$, almost $60 \%$ of AKI occurred in patients who never received care in an intensive care setting. While we cannot ascertain if AKI reflected a primary condition or acted as a marker of severity of illness, or whether these patterns reflect clinical practices at this center, we surmise that earlier case or acuity detection might improve the care of these individuals. We also observed that in some patients AKI occurred after 7 days of hospitalization, and that mortality was higher in this subset. The latter finding suggests that initial hospital care often fails to prevent AKI.
We identified a spectrum of conditions susceptible to $\mathrm{AKI}$, including cardiovascular disease, infections and sepsis, complications from medical care, neoplasms, and injury. Furthermore, the odds of mortality varied across disease groups. These observations are important because AKI associated with different conditions may require different management strategies. For example, AKI associated with trauma may be related to rhabdomyolysis, pancreatitis, and/or respiratory failure. AKI associated with neoplasms may reflect acute toxicity from chemotherapeutic agents or antibiotics used to treat infection. We expect that the delineation of the etiology is an important step for defining AKI prevention and treatment strategies.

\section{Limitations}

Only $40 \%$ of hospitalizations in this series received two or more $\mathrm{sCr}$ measurements; the incidence of $\mathrm{AKI}$ is unknown in the remaining $60 \%$ of inpatients. While these individuals may have had a lower severity of illness, more frequent sCr testing across all patients may have increased the number of detected AKI cases. We described patients who developed AKI in the hospital setting but did not describe those patients who had already developed AKI prior to hospital admission. We note that the subsequent course of these patients may be variable, with some patients improving and others exhibiting additional increases in $\mathrm{sCr}$. Despite this limitation, we still identified substantial AKI incidence rates, which may have been even higher if we had had access to baseline sCr levels [17, 18].

Our data originated from a single medical center, and extrapolation to the entire US hospitalization should be made with caution. The replication of these findings in other academic or community hospital settings would further strengthen the validity of the conclusions; the applicability of the UHC global mortality estimator could facilitate these comparisons. We did not have access to physiological variables such as heart rate, systolic blood pressure, oxygenation, or temperature, a common limitation of administrative data. In addition, we did not have detailed information on the care received prior to hospital presentation (for example, from paramedics). 


\section{Conclusion}

In the current study, AKI occurred in over 1 of 5 hospitalized patients and was associated with an over fourfold increased mortality - findings that appear to persist over the last decade. These observations offer new insights into the current epidemiology of AKI and highlight the continued need to improve the detection of AKI and reduce the incidence and consequences of AKI.

\section{Acknowledgements}

We are grateful to Darlene Green and Stephen Duncan, who provided the data set and technical guidance. Dr. Wang received grant support from award R01-NR012728 from the National Institute of Nursing Research.

\section{Disclosure Statement}

The authors declare that they have no conflict of interest to disclose.

\section{References}

1 Chertow GM, Burdick E, Honour M, Bonventre JV, Bates DW: Acute kidney injury, mortality, length of stay, and costs in hospitalized patients. J Am Soc Nephrol 2005;16: 3365-3370.

2 Uchino S, Bellomo R, Goldsmith D, Bates S, Ronco C: An assessment of the RIFLE criteria for acute renal failure in hospitalized patients. Crit Care Med 2006;34:1913-1917.

> Nash K, Hafeez A, Hou S: Hospital-acquired renal insufficiency. Am J Kidney Dis 2002; 39:930-936.

4 Liangos O, Wald R, O’Bell JW, Price L, Pereira BJ, Jaber BL: Epidemiology and outcomes of acute renal failure in hospitalized patients: a national survey. Clin J Am Soc Nephrol 2006;1:43-51.

5 Waikar SS, Curhan GC, Ayanian JZ, Chertow GM: Race and mortality after acute renal failure. J Am Soc Nephrol 2007;18:27402748.

-6 Bellomo R, Ronco C, Kellum JA, Mehta RL, Palevsky P: Acute renal failure - definition, outcome measures, animal models, fluid therapy and information technology needs: the Second International Consensus Conference of the Acute Dialysis Quality Initiative (ADQI) Group. Crit Care 2004;8:R204R212.
$>7$

Mehta RL, Kellum JA, Shah SV, Molitoris BA, Ronco C, Warnock DG, Levin A: Acute Kidney Injury Network: report of an initiative to improve outcomes in acute kidney injury. Crit Care 2007;11:R31.

$>8$ Ricci Z, Cruz D, Ronco C: The RIFLE criteria and mortality in acute kidney injury: a systematic review. Kidney Int 2008;73:538-546.

-9 Bagshaw SM, George C, Bellomo R: Early acute kidney injury and sepsis: a multicentre evaluation. Crit Care 2008;12:R47.

10 Liborio AB, Abreu KL, Silva GB Jr, Lima RS, Barreto AG, Barbosa OA, Daher EF: Predicting hospital mortality in critically ill cancer patients according to acute kidney injury severity. Oncology 2011;80:160-166.

11 Lopes JA, Fernandes P, Jorge S, Resina C, Santos C, Pereira A, Neves J, Antunes F, Gomes da Costa A: Long-term risk of mortality after acute kidney injury in patients with sepsis: a contemporary analysis. BMC Nephrol 2010;11:9.

12 Uchino S, Kellum JA, Bellomo R, Doig GS, Morimatsu H, Morgera S, Schetz M, Tan I, Bouman C, Macedo E, Gibney N, Tolwani A, Ronco C: Acute renal failure in critically ill patients: a multinational, multicenter study. JAMA 2005;294:813-818.
13 Levey AS, Stevens LA, Schmid CH, Zhang YL, Castro AF 3rd, Feldman HI, Kusek JW, Eggers P, Van Lente F, Greene T, Coresh J, CKD-EPI (Chronic Kidney Disease Epidemiology Collaboration: A new equation to estimate glomerular filtration rate. Ann Intern Med 2009;150:604-612.

14 Mortality Risk Adjustment Methodology for University Health System's Clinical Data Base, available at http://www.ahrq.gov/qual/ mortality/Meurer.htm (accessed September $16,2011)$.

15 Angus DC, Linde-Zwirble WT, Lidicker J, Clermont G, Carcillo J, Pinsky MR: Epidemiology of severe sepsis in the United States: analysis of incidence, outcome, and associated costs of care. Crit Care Med 2001;29: 1303-1310.

16 Wang HE, Shapiro NI, Angus DC, Yealy DM: National estimates of severe sepsis in United States emergency departments. Crit Care Med 2007;35:1928-1936.

17 Lafrance JP, Miller DR: Defining acute kidney injury in database studies: the effects of varying the baseline kidney function assessment period and considering CKD status. Am J Kidney Dis 2010;56:651-660.

18 Ali T, Khan I, Simpson W, Prescott G, Townend J, Smith W, Macleod A: Incidence and outcomes in acute kidney injury: a comprehensive population-based study. J Am Soc Nephrol 2007;18:1292-1298. 